\title{
A design for Intelligent Parking System Based on ETC Technology and Android
}

\author{
Xiaomin Wang \\ Department of Electronic Engineering \\ Tianjin University of Technology and Education \\ Tianjin, China \\ Email: xiaominwang111@126.com
}

\author{
Zhijun Pei \\ Department of Electronic Engineering \\ Tianjin University of Technology and Education \\ Tianjin, China \\ email:teacherpei@126.com
}

\begin{abstract}
With the rapid growth of private car ownership in the city, parking is difficult in the residential area and chaotic parking problems have become increasingly prominent. In view of the phenomenon, this paper makes electronic toll collection technology as the core design of a set of residential parking on intelligent parking management system based on the Android platform. The system enables ETC technology, embedded technology, streaming video conversion technology, wireless sensor technology, 3G/4G network technology rolled into one. After the simulation and testing, this system is more efficient and convenient than traditional parking system, has a high value.
\end{abstract}

Keywords- ETC; Intelligent Parking; RSU; DSRCAndroid

\section{INTRODUCTION}

With the rapid development of economy and the continuous improvement of people's living standard, private car ownership is growing fast in China. The phenomenon of difficult parking and chaotic parking problems are becoming more and more serious. The owners have to install parking lock in order to safeguard their legitimate rights and interests and make theirs own car stop immediately at any time. However, parking lock also brings about a series of inconveniences. For example, in order to make their cars into the right place, owners have to get out of cars and do some manual operations. Even remote parking lock can bring many problems, such as remote control. Plus cars in and out of plot often concentrated in the rush hour, the village guards cannot open the entrance guard system rail vehicle because of time saving at random. Besides, it is unrealistic to check any car, which brings great inconvenience to owners. In view of the problems above, this paper will introduce ETC technology into community intelligent parking management system, and make the vehicle from through checking the door to the parking lock automatically opened, and other functions.

\section{BRIEF INTRODUCTION OF ETC TECHNOLOGY}

ETC technology, one of the most important part of recent Intelligent Transportation System(ITS), just keeps the vehicle at a speed of $20 \mathrm{~km} / \mathrm{h}$ through charging entrance without stopping to finish vehicle identification, automatic charging and so on ${ }^{[1]}$.ETC technology is more efficient and convenient compared with traditional Manual Ton Collection system (MTC). ETC mainly consists of On-Board Unit (OBU), IC card, Road - Side Units (RSU), lane controller, financial management systems and computer auxiliary equipment (such as camera system and infrared detection system) ${ }^{[2]}$. Working principle of ETC is shown as Fig. 1.

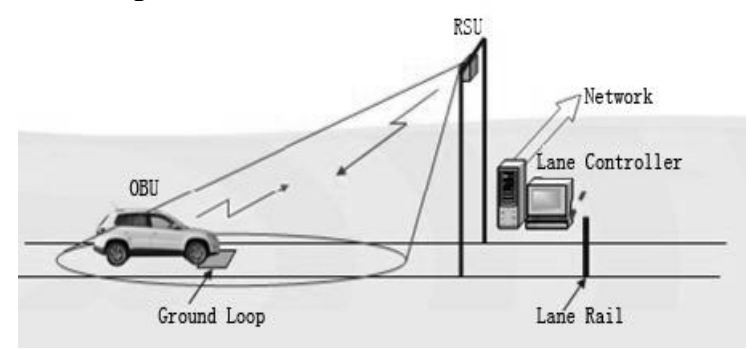

Figure 1. Working principle of ETC

\section{OVERALL DESIGN OF THE SYSTEM}

Intelligent parking system is divided into five parts, OBU, access control unit, parking lock unit, security unit, and Android platform.

Community access control RSU establishes microwave communication link with car OBU through Dedicated Short Range Communications (DSRC). The aim is to realize vehicle identification and decide whether to release through wire less remote control to open parking lock automatically without a stop, achieve intelligent parking eventually. OBU supplied by batteries must take selective opening/closing operations to extend battery replacement cycle for transmission circuit. Grand loop mounted on the front of access control is used to arouse OBU transmission circuit. OBU transmission circuit is dormant when the car is moving. Grand loop will detect car signal and open transmission circuit automatically when the car through it. This realizes two-way communication between vehicles and access control ${ }^{[3]}$. ETC systemstructure is shown in Fig. 2.

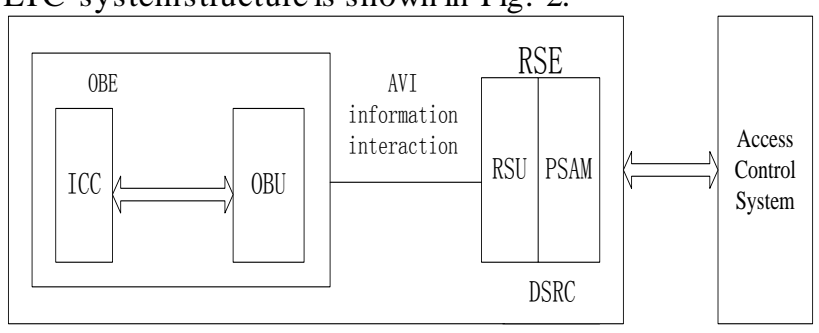

Figure 2. ETC system structure 
Communication between access control and the car is DSRC technology ${ }^{[4]}$.DSRC technology is mainly used in the field of ITS short distance wireless communication protocols, including three parts: RSU, OBU and DSRC protocol. DSRC protocol structure consis ts of three layers, namely PHY (Physical Layer), LLC (Data Link Layer) ${ }^{[5]}$, and Application Layer.

MAC layer controls the use of RSU and OBU wireless channel, providing the physical interface and validation of the received frame is in line with the regulations. Equipments above Application layer are based on DSRC, during which the communication is done through the service primitives. The frequency is $5.8 \mathrm{GHz}$, output power is $300 \mathrm{~mW}$, communication distance is $10 \sim 30 \mathrm{~m}$, modulation mode to ASK ${ }^{[6,7]}$. RSU generally does not write only read to OBU devices. DSRC data flow diagram is shown in Fig. 3.

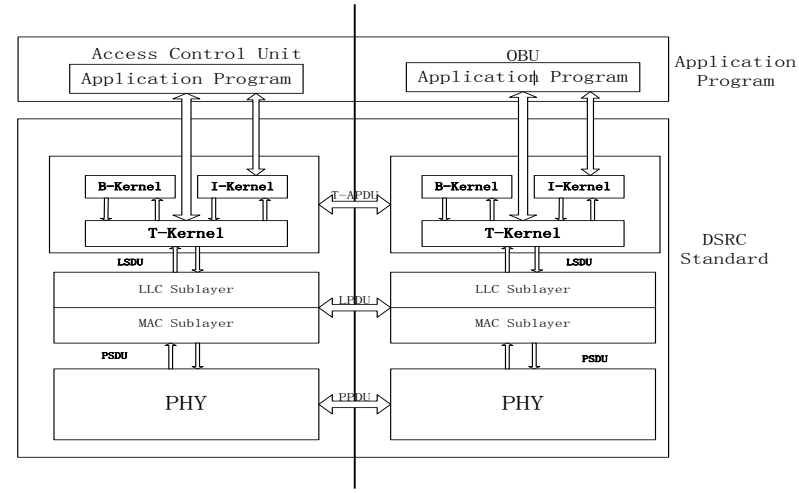

Figure 3. DSRC data flow diagram

Security unit takes video monitoring as the core, combining with the Android platform to form "mobile phone security" module on the basis of the Android platform. Using mature $3 \mathrm{G} / 4 \mathrm{G}$ network to achieve realtime mobile video monitoring. To realize user login, view parking occupancy, open/close security monitoring, open/close parking lock, and modify information in community service system through Android client.

\section{THE REALIZATION OF HARDWARE}

\section{A. Access control unit}

Access control unit takes a core position in the system, not only decide whether to release vehicles into the community, however, also control whether to lock or open parking lock. Community access control unit consists of MCU (Micro Control Unit), encoding radio frequency, interface communication and display screen. MCU is the core of hardware, and almost all of the functions including sending and receiving signals, opening the entrance guard rail, controlling the parking lock and the display screen, need to be controlled by the MCU to complete. The overall structure of access control system is shown in Fig. 4.

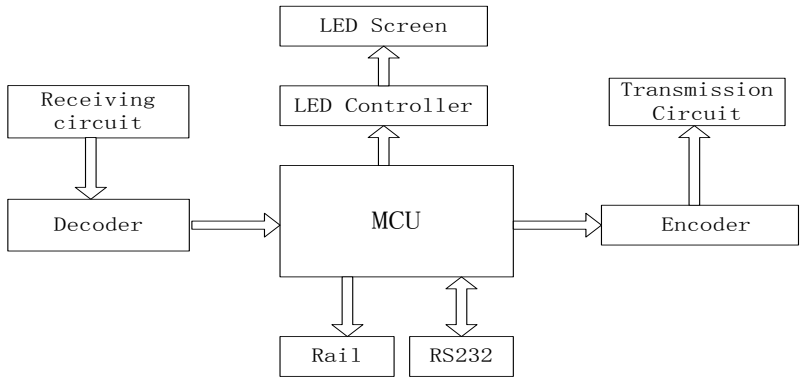

Figure 4. Overall structure of access control system

MC9S08DZ60 produced by Freescale Company is chosen as chip of MCU part. The frequency of MC9S08DZ60 is $40 \mathrm{MHZ}$, and the storage space is as high as $60 \mathrm{~K}$. The maximum space of EEPROM online programmable internal memory can reach $2 \mathrm{~K}$. As to system protection, a watchdog (COP) reset, illegal operation address reset, illegal code reset, and other functions have been equipped. In terms of power-saving, MC9S08DZ60 provides two types of power-saving modes: one is a waiting mode with reducing power consumption, and the other is a break mode with ultra-low power and real time clock (can operate under all modes including operation modes, waiting modes and stopped modes). There are 53 general I/O pins and one dedicated input pin. The device pins assignment is shown in Fig. 5.

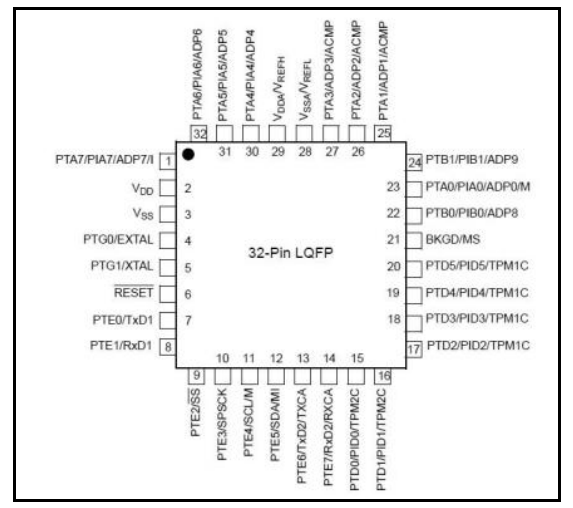

Figure 5. MC9S08DZ60 32pin LQFP

When the receiving circuit receives signals from the on-board unit, it will decode and send the signals to MCU through PTE1 / RxD1 port to process. MCU calls detect_signal () function to judge whether the vehicle belongs to this community and decide whether to release according to the judging results. Opening/closing entrance guard rail is controlled by PTB0 / PTB 1 ports. At the same time, the LED display is controlled by PTE6 / PTE7 ports and LED controlling module. Open timer, and 10s later (to prevent the parking areas are occupied by other cars before entering. You can make appropriate adjustments according to the actual situation of geographical environment) launch the corresponding signal of opening the parking lock. During the process, transmitting signals is performed by PT2262 controller chip in PTA port. The transmission circuit is made up of a high frequency oscillator (315 MHZ, carrier signal) and a low frequency oscillator (modulation signal). 


\section{B. On-Board Unit}

OBU consists of the following modules: MCU module, power management module, wireless radio frequency module and memory module.

MSP430F5418A which is produced by TI (Texas Instruments) company is chosen as the MCU master control chip. PD5200 produced by a South Korea company Phychips is chosen as transceiver chip of wireless if module selection. This chip has a high integrated level, and thus can simplify the design of the circuit structure. PD5200 have integrated with the wire less awaken module inside, so we needn't design extra external wireless awaken module, and this integration also plays a role in saving power. PD5200 has four working modes: PD_MODE, WK_MODE, RX_MODE, TX_MODE. The wireless trans mitting module is controlled by MCU control module MSP430F5418A together with PD5200.

When PD5200 works in WK_MODE mode, only the internal wireless awaken module is in working condition. When awaking signal from RSU unit is tested, the interrupt signal will be sent to MSP430F5418A through WK_DT port. PD5200 is controlled by MSP430F5418A through TX_EN and RX_EN port. MCU controls PD5200 through TX - EN, and PD5200 sends TX - ENb which is generated through PD5200 internal logic control to SPDT. $\mathrm{TX}-\mathrm{EN}$ is contrary to $\mathrm{TX}-\mathrm{ENb}$ level signal, therefore, sending and receiving signals can be controlled finally. The specific wave form of waking up signal is processed by PD5200 internal register SE_WK_DATA. The working principle of radio frequency emission module is shown in Fig. 6. MSP430F5418A has rich interface resources. Interfaces of MSP430F5418A and PD5200 are shown in Fig. 7.

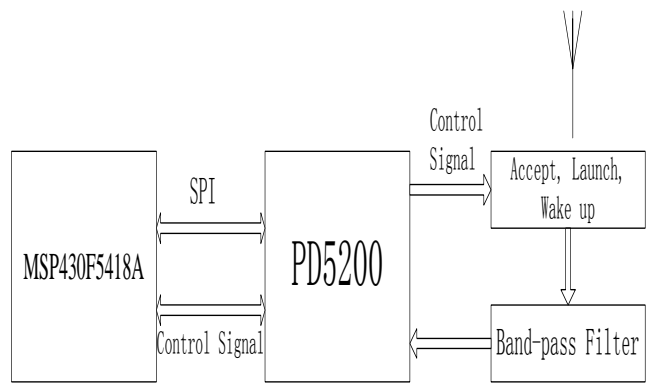

Figure 6. Working principle of radio frequency emission module

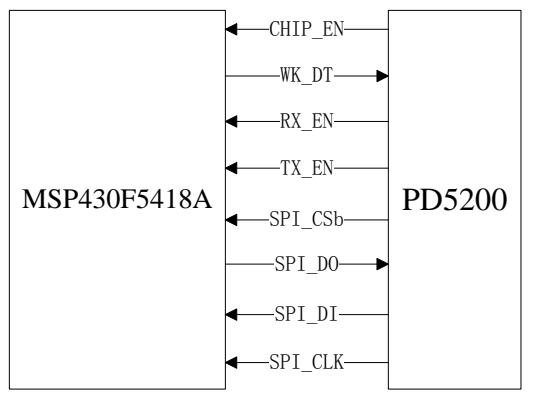

Figure 7. Interfaces of MSP430F5418A and PD5200

OBU reads the information on the IC card and sends signals to RSU through RF circuit. IC card is mainly used to store information about vehicles, such as license plate number, models and corresponding parking number inside the residential area, etc. The OBU communicate with RSU through the DSRC. When vehicles go through groundsense coil, RSU will send signals to awake OBU to send signals. If both sides confirm the signals, then the vehicle will be released and the parking lock will also be open.

\section{Parking lock unit}

Parking lock unit can automatically open/close the parking lock by receiving wireless signals sent by entrance guard unit. Parking lock hardware can be divided into: MCU module, power module, wireless receiving module and motor control module according to different functions. Since the parking lock motor power supply and MCU power supply module have different requirements on voltage, different batteries are used respectively. Parking lock motor is powered by a $12 \mathrm{v}$ battery, while the embedded power supply module adopts a $5 \mathrm{v}$ battery. AT89C51 is used by MCU parts. Decoding part employs PT2272, which adopts CMOS technology on the chip, and has low power consumption. Less external elements and the eight address code. The number of the address code that does not reduplicate comes to 6561 , which can meet the requirements of residential parking system quite well. Only when the address code sent by the decoder (PT2272) is totally same with the address code which is launched by the entrance guard unit encoder module (PT2262), will the system send interrupt signal to $\mathrm{MCU}$ unit to control the rise and fall of parking lock. The working principle of the parking lock is as following in Fig.8.

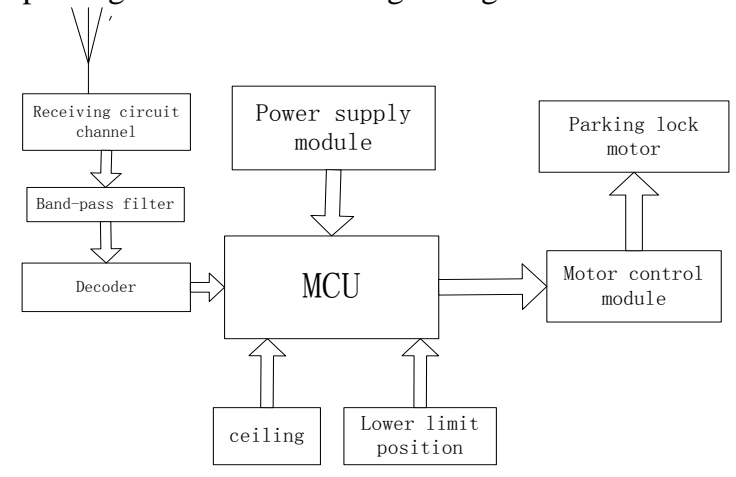

Figure 8. Working principle of parking lock unit

The rotation of the lifting arm is controlled by the rotation of the motor. When the lifting arm rise/fall to the specified position, the motor will stop rotation because of screens function of the mechanical node control, and thus the equipment can be protected. Motor control module includes: signal input, the positive and negative power supply monitoring, current limiting circuit, Vbe monitoring, etc.

\section{THE REALIZATION OF SOFTWARE}

The realization of software adopts the thought of modular, it can not only improve the portability of the program, However, also it is convenient for the system to upgrade in the future.

\section{A. Access control program design}

Access control unit is not only responsible for communication with OBU and confirmation, and it will be responsible for communication with parking lock. 
Software design of access control unit includes: communication module, control module and display module. After MC9S08DZ60 MCU chip's initialization, through ground loop coil level's transform, it detects whether there are vehicles through the access control. When vehicles were observed through the access control, access control and OBU co mmunication will co mp lete the confirmation of vehicles, to decide whether to open the rail and open the corresponding parking lock.

Access control unit is not only responsible for communication with $\mathrm{OBU}$ and confirmation, but also responsible for communication with parking lock. Software design of access control unit includes: communication module, control module and display module. After MC9S08DZ60 MCU chip's initialization, through ground loop coil level's transform, it detects whether there are vehicles through the access control. When vehicles were observed through the access control, access control and OBU communication will co mp lete the confirmation of vehicles, to decide whether to open the rail and open the corresponding parking lock.

The process of MCU initialization needs initialization functions include: IO interface initialization (int_IO ()), LED display control initialization (int_LED ()), MCU initialization configuration information initialization (int_configuation ()) and interrupt initialization (int_interrupt ()).

The vehicle information is stored in the form of an array. Car distribution in the system ID number is the serial number of the array, calling function check_car, making confirmation on the vehicle () function. ID is able to locate the position of the vehicle information in the array directly and quickly, in an efficient way. Vehicle information storage format is: \{ID number +car license + plate number $\}$.For example $\{105$, jin A1P996, $1105\}$. Software design flow chart of access control unit is shown as Fig. 9.

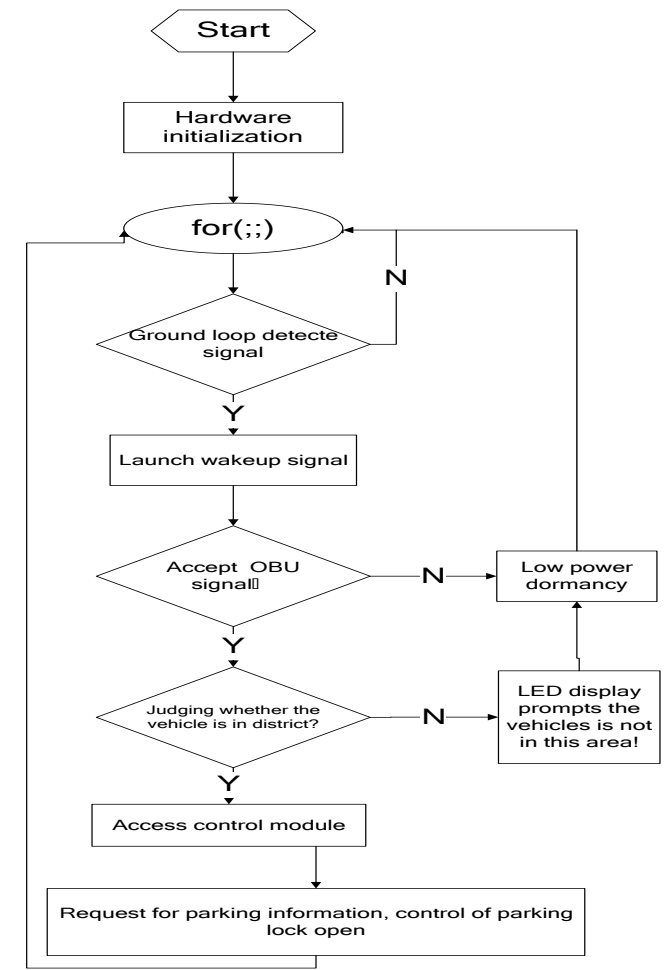

Figure 9. Software design flow chart of access control unit

\section{B. OBU program design}

$\mathrm{OBU}$ is mainly responsible for RF signal sending, receiving and for MCU's encoding and decoding of signals. On-board unit of software system mainly includes: MCU control, DSRC protocol processing and management of power supply module. The DSRC protocol processing is the core part of the system. It is mainly responsible for processing of data between the parameters of each layer, data frames, communication links and various kernels. The division of the OBU software function is shown in Fig. 10.

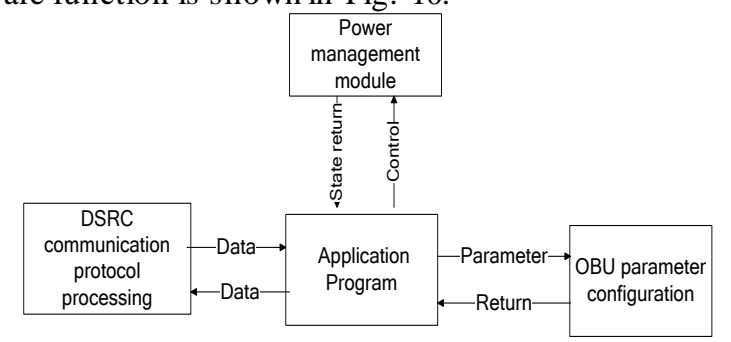

Figure 10. The division of the OBU software function

OBU radio frequency module detects RSU unit wake signal, PD5200 port WK_DT generate the interrupt signal to MSP430F5418A.The system consists of a dormant state into running state. The software flow chart is shown in Fig. 11. 


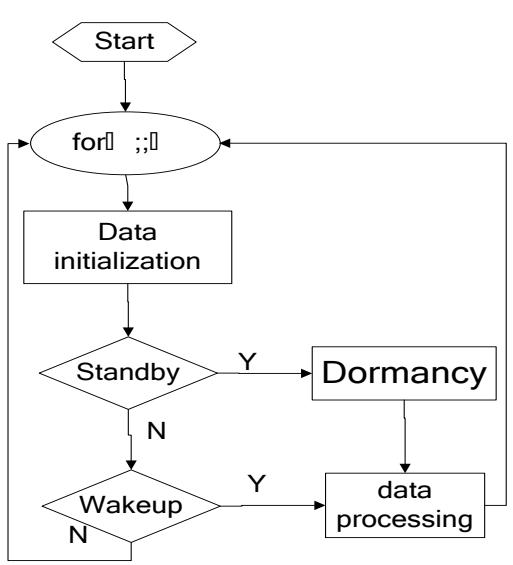

Figure 11. The MCU control flow chart of OBU

\section{Parking locking program design program design}

Parking lock is mainly responsible for accepting wireless signal from access control unit. Signal will eventually be transmitted to MCU through band-pass filter and decoders decoding. MCU will make corresponding processing after receiving the signal.

The main () function of parking lock initialization program includes: interrupt initialization (external interrupt, edge interrupt, opening a serial port interrupt and global interrupt), clock initialization, serial port initialization void UART_int (void), etc. After the initialization process, it will enter infinite loop for (;;), detecting interrupt signal from the decoder, and control the motor rotation, finally completes the automatic parking. The software flow chart is shown in Fig. 12.

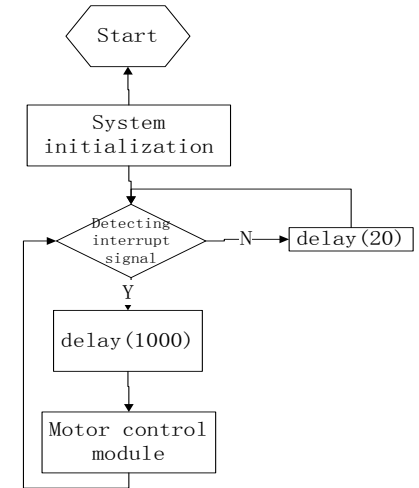

Figure 12. Software flow chart of parking lock

\section{SECURITY UNIT DESIGN}

Security unit combines the traditional video technology with modern network communication technology, the formation of video technology, 3G / 4G network technology, and the video monitoring system combines with the Android platform. With $4 \mathrm{G}$ networks' (e.g. mobile $4 \mathrm{G}$ download speeds up to $100 \mathrm{MBPS}$ ) gradual popularization, mobile video data stream transmission is no longer bound by wireless bandwidth bottlenecks. With the support of high-speed wireless network, customers can use a mobile phone anytime, anywhere to get control of monitoring site. Because video formats supported by Android are very limited (such as: 3 gp and MPEG4, etc.). What needs to solve in this article is changing ASF flow that collected by camera into MPEG4 format supported by the Android platform through conversion tool VLC (Video Lan Client), Android will parse MPEG4 format and output RTSP stream. Realize monitor screen display control through MediaPlayer and implement the play function through SurfaceView. Set configure file to close some unnecessary functions of VLC and open the necessary function ${ }^{[} 8,9^{]}$. Video format conversion is shown in Fig. 13.

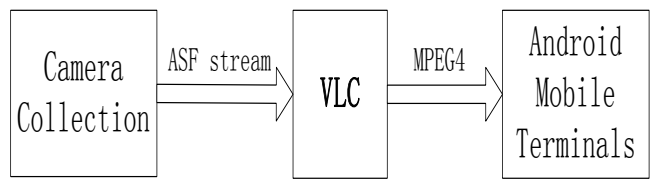

Figure 13. Video format conversion

\section{ANDROID PLATFORM DESIGN}

Android system of smart phone market share is so high because the openness of the Android and the clear of man-machine interface, so we choose Android as the system client software development platform. Mobile terminal software is composed of accessing the interface and main menu interface. Creating MenuActivity class and inheriting Activity class make MenuActivity class owns the property of the Activity, autotype onCreat () method. Define Button class variables bt_theme, bt_lock, bt_jiankong, bt_set and bt_help respectively represent software Settings, parking lock switch, the theme of the monitor Button, parameter Settings and help information. The implementation of the code is simpler so we don't talk detailed. User information is stored in the Android built-in database SQLite. To embed the video playback function of security unit in the client APP. Considering the actual situation of basic security video image tend to be fixed, we can choose another mode: refresh the mobile terminal in a certain cycle video images, thus reducing the data traffic. Connection-oriented Socket nested technology is adopted to realize the communication between the Android mobile terminal and server. Fig. 14 is a part screenshot about Android APP interface.

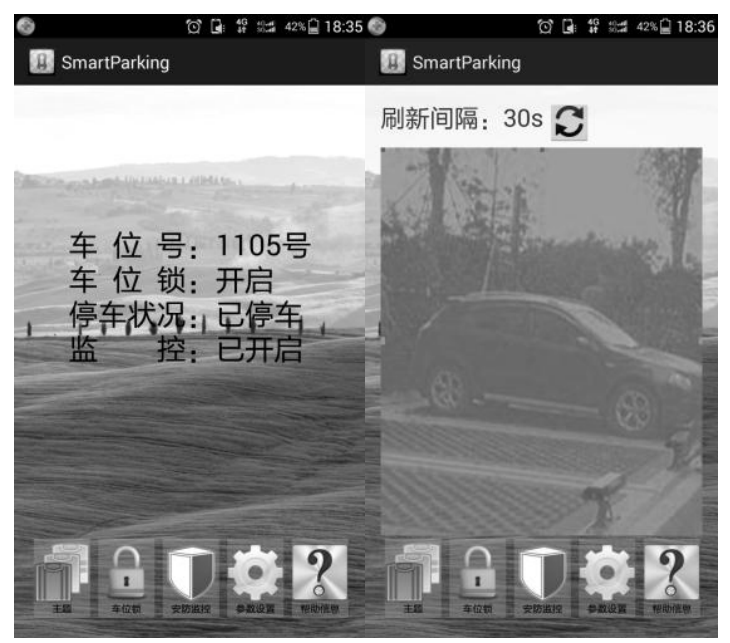

Figure 14. A part screenshot about Android APP interface 


\section{CONCLUSIONS}

ETC technology in ITS is introduced into the intelligent parking system in this paper, which realizes the process of automatic parking from community access control confirm to parking lock open intelligence and through the "mobile phone video surveillance" at anywhere to know the monitoring field. This design is based on Android platform mobile client, it is simple, and at the same time achieves the function of remote monitoring, which has a higher application value.

\section{ACKNOWLEDGMENT}

This work is supported by Research Foundation of Tianjin University of Technology and Education under Grant No. KJY1312 and Innovation Fund for Graduate Students of TUTE under Grand No.YC14-06.

\section{REFERENCES}

[1] XIU Hua-wei, ZHANG Nan, ZHANG Hai-yan. Application of ETC Technology in Underground Parking [J].System Computer \& Digital Engineering, 2009,37(5) : 103-105.

[2] JIANG Wei, ZHAO Rong-hua, LI We. The Application of Dedicated Short Range Communication in Intelligent
Transportation System[J]. Journal of Telecom Science, 2001,17(8):14-16.

[3] TU Yu, XU Jian-min, ZHONG Hui-ling. Development of Communication Protocol for DSRC system [J]. Computer Engineering, 2003,29(21):28-30,33.

[4] FAN Qing-bin, SUN Jian-song, MU Hong-guang. Study on the DSRC technology and Communication Mechanism [J]. Journal of telecom science, 2010,26(8):99-101.

[5] CHEN Ling-xu, ZHANG Cui-fang, LI qiang. State analysis of logic link control Layer of dedicated short range communication protocol[J]. Journal of Transportation Engineering and Information, 2005, 3 (2): 68-73.

[6] WANG Xin. Research and Design of the RSU and Roadway controller in the ETC System Based on the DSRC Protocol [D].Shanxi: Xian university of electronic science and technology,2008.

[7] ZHANG Di. Design and implementation of OBU side DSRC protocol in ETC system Shandong: Shandong University, 2009.

[8] XIONG Guang-hui. Design and implementation of mobile remote monitoring system based on Android platform [D].Sichuan: University of Electronic Science and Technology of China, 2012.

[9] LI Ning. Video surveillance system design on Android [D]. Beijing :Beijing university of posts and telecommunications, 2011.

[10] LI Qin, CHEN Li-ding, REN Zhi-gang. Design of Remote Video Monitoring System Based on Smart Phone Android Platform[J]. Video Engineering,2012,36(7):134-136. 